\title{
Assembly and immunological processing of polyelectrolyte multilayers composed of antigens and adjuvants
}

Yu-Chieh Chiu, $†$ Joshua M. Gammon, $†$ James I. Andorko, † Lisa H. Tostanoski, $†$ and Christopher M. Jewell*,†, $\ddagger$,

$\dagger$ Fischell Department of Bioengineering, University of Maryland, College Park, 8228 Paint Branch Drive, Room 2212 Jeong H. Kim Building, College Park, MD, 20742, United States

¥ Department of Microbiology and Immunology, University of Maryland Medical School, 685 West Baltimore Street, HSF-I Suite 380, Baltimore, MD, 21201, United States

$\S \quad$ Marlene and Stewart Greenebaum Cancer Center, 22 S. Greene Street, Suite N9E17, Baltimore, MD 21201, United States

* To whom correspondence should be addressed: Prof. Christopher M. Jewell, Fischell Department of Bioengineering, University of Maryland, 8228 Paint Branch Drive, Room 2212 Jeong H. Kim Building, College Park, MD 20742. Office: 301-405-9628, Fax: 301405-9953, E-mail: cmjewell@umd.edu, Web: jewell.umd.edu 


\section{SUPPORTING FIGURES AND LEGENDS}
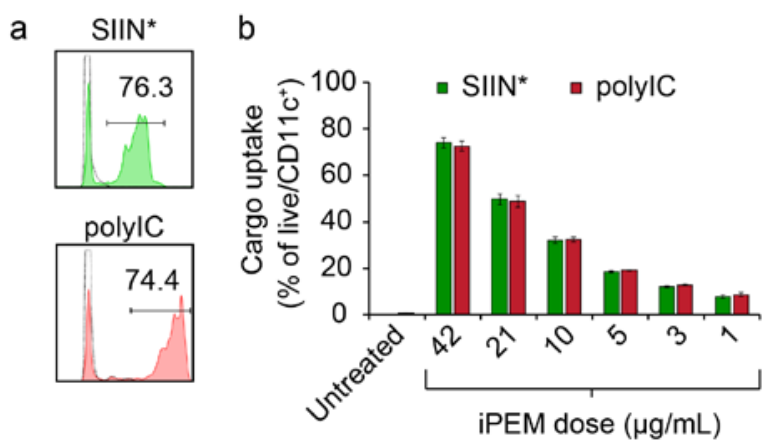

Figure S1. iPEM capsules are internalized by primary DCs. a) Representative histograms demonstrating SIIN* and polyIC uptake during treatment of splenic DCs with (SIIN*/polyIC) 3 iPEM capsules for 24 hrs. b) Aggregate analysis of dose dependent uptake of (SIIN*/polyIC) 3 iPEMs. Values indicate the mean \pm s.e.m. SIIN* represents SIINFEKL-Rg. 


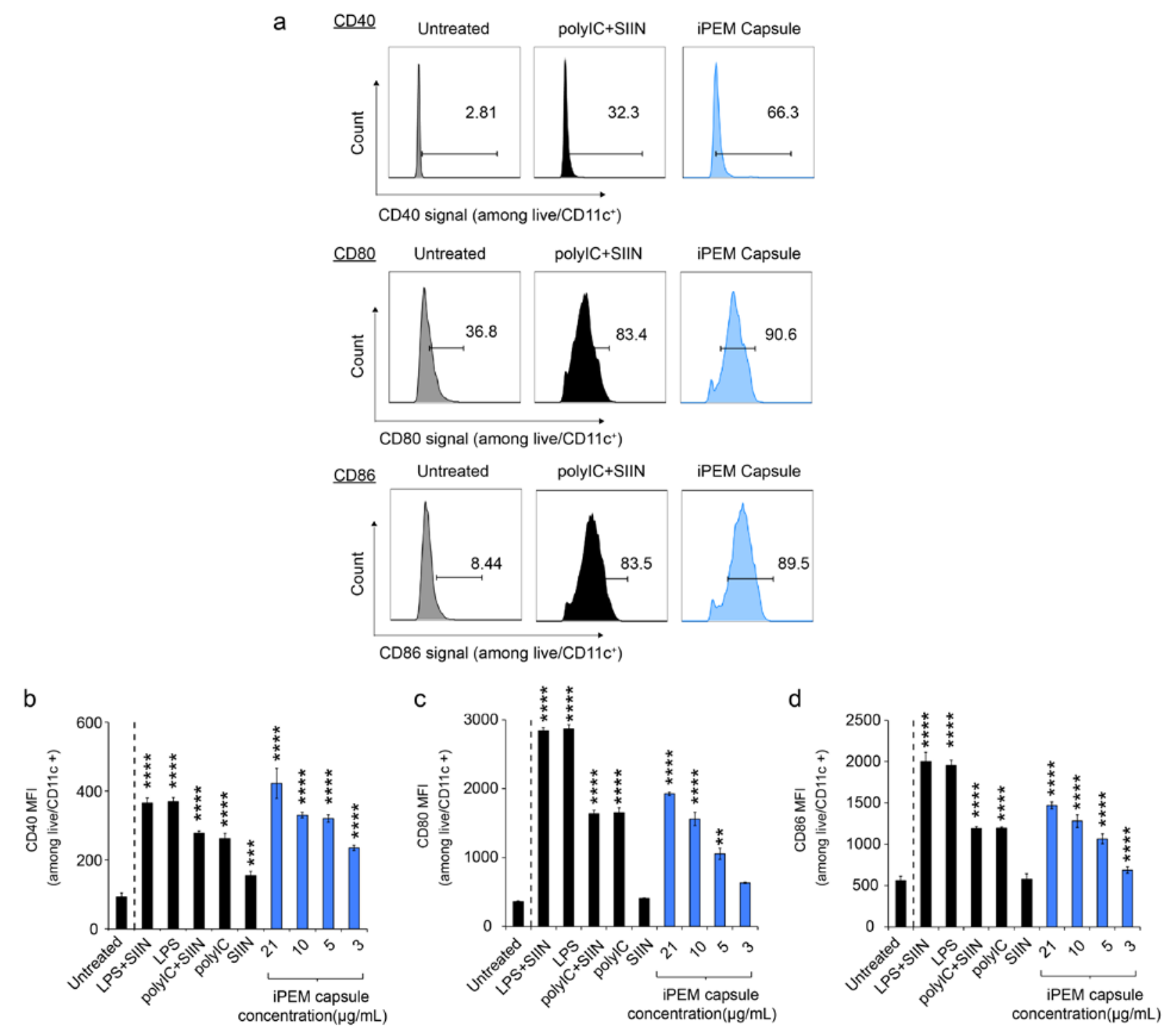

Figure S2. In vitro activation of DCs by iPEM capsules. a) Representative flow cytometry histograms of CD40, CD80, and CD86 for samples that were untreated, treated with polyIC (10 $\mu \mathrm{g} / \mathrm{mL})+$ SIIN $(5 \mu \mathrm{g} / \mathrm{mL})$, or treated with iPEM capsules. b-d) Mean fluorescent intensities (MFIs) of (b) CD40, (c) CD80, and (d) CD86 expression after treatment with iPEM capsules for 24 hrs. Values for all panels indicate the mean \pm s.e.m. Statistical comparisons indicate significance of $* *=p \leq 0.01, * * *=p \leq 0.001$, and $* * * *=p \leq 0.0001$, and for clarity, are shown for each group compared against the control (Untreated; dashed line). SIIN represents SIINFEKL. 

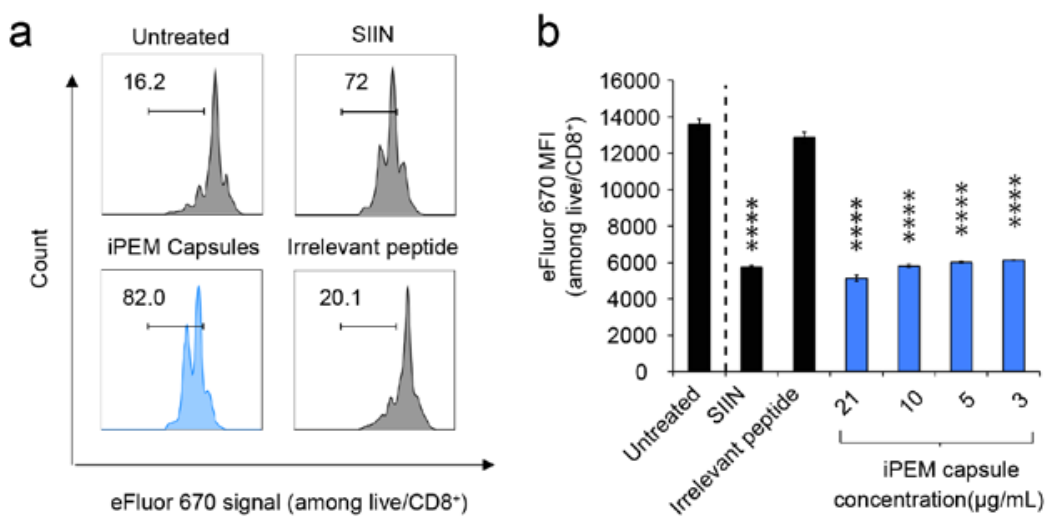

Figure S3. iPEM capsules drive antigen-specific T cell expansion in vitro. a) Representative histograms showing the frequency of proliferated OT-I CD8 ${ }^{+} \mathrm{T}$ cells after co-culture with untreated DCs or DCs treated with SIIN $(5 \mu \mathrm{g} / \mathrm{mL})$, irrelevant peptide $(5 \mu \mathrm{g} / \mathrm{mL})$, or iPEM capsules $(21 \mu \mathrm{g} / \mathrm{mL})$. Proliferation was quantified using a standard fluorescence proliferation dye (eFluor 670) after 48 hrs. b) MFIs of fluorescent dye used to measure proliferation of $\mathrm{CD}^{+} \mathrm{T}$ cells analyzed by flow cytometry. Values for all panels indicate the mean \pm s.e.m. Statistical comparisons indicate significance of $* * * *=p \leq 0.0001$, and for clarity, are shown for each group compared against the control (Untreated; dashed line). SIIN represents SIINFEKL. 